\title{
基于叶片气孔导度提升的冬小麦冠层阻抗估算模型的 应用和对比
}

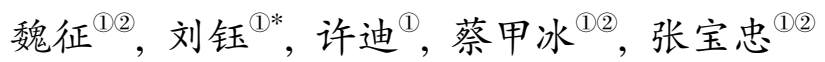

(1) 中国水利水电科学研究院, 流域水循环模拟与调控国家重点实验室, 北京 100038;

(2) 国家节水灌溉北京工程技术研究中心, 北京 100048

*联系人, E-mail: liuyu@iwhr.com

2012-11-19 收稿, 2013-03-28 接受

国家重点基础研究发展计划(2006CB403405)、水利部“948”项目(201009)和国家自然科学基金(91125017, 51109225, 51009151, 50909099)资助

\begin{abstract}
摘要 为了篮选适合华北典型地区冠层阻抗基于叶片气孔导度的提升方法, 依据两个生长季 的冬小麦田间试验资料, 以 Leuning-Ball 和 Jarvis 叶片气孔导度模型为基础, 构建了冠层阻抗 估算模型, 对比了不同冠层阻抗估算模型的应用效果. 结果表明: 冬小麦叶片气孔阻抗日变化 整体高于冠层阻抗, 两者具有显著的尺度差异性; Leuning-Ball 气孔导度模型能够更好地解释 气孔导度对环境因子的响应; 以光合有效辐射作为尺度转换因子, 基于 Leuning-Ball 气孔导度 模型提升的冠层阻抗估算模型能够有效地模拟冠层阻抗，可应用于华北典型地区冬小麦水汽 传输阻抗尺度提升研究.

关键词

模型

辐射

叶片气孔导度

冠层阻抗

尺度提升

冬小麦
\end{abstract}

蒸散是植物群体与外界环境物质、能量交换的一 个重要组成部分, 其准确估算对于理解水碳耦合、物 质能量循环具有重要的意义. 蒸散的模型有单层模 型 ${ }^{[1]}$ 、双层模型 ${ }^{[2]}$ 和多层模型 ${ }^{[3]}$ 等, 这些模型均是通过 不同的阻抗来描述土壤蒸发和植株蒸腾. 尽管冠层 阻抗 $\left(r_{\mathrm{c}}\right)$ 已经用于计算参考作物蒸发蒸腾量和实际作 物蒸发蒸腾量中, 但是目前没有不同作物在不同生 育阶段冠层阻抗 $\left(r_{\mathrm{c}}\right)$ 的推荐值 ${ }^{[4]}$, 冠层阻抗 $\left(r_{\mathrm{c}}\right)$ 在不同 环境下的可用性决定蒸散的精度. 因此, 可靠的冠层 阻抗 $\left(r_{\mathrm{c}}\right)$ 估算成为一个非常重要的研究课题, 其中基 于叶片气孔导度向冠层阻抗提升的估算模型则是该 研究中的热点.

基于叶片气孔导度向冠层阻抗(或冠层导度)提 升的研究中, 一是在采用气孔计、光合仪等设备直接 测定作物不同叶位的叶片气孔导度数据基础上, 利 用整体平均法、顶层阳叶分层采样法、权重法、有效 叶面积指数法、水平冠层分层法、多冠层叶倾角分类

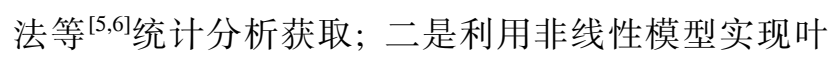
片气孔导度向冠层阻抗转换的尺度提升, 其中一些 方法把叶片尺度到冠层阻抗的提升模型视为不同的 气象、土壤以及作物生长特性的函数 ${ }^{[7 \sim 9]}$, 如 Jarvis 叶片气孔导度模型在冠层阻抗 $\left(r_{\mathrm{c}}\right)$ 估算中的应用, 但 是 Jarvis 模型多是基于短时期的观测资料, 讨论冠层 阻抗 (导度)对叶温、饱和差、光合有效光量子密度、 叶水势响应函数, 及多变量函数乘合的冠层阻抗(导 度)模型的最优化方法 ${ }^{[10]}$, 和长时间尺度条件下, Jarvis 模型的应用性和适用性 ${ }^{[11]}$. 另一些方法则是从环 境和能量平衡的角度推出尺度提升模型 ${ }^{[12,13]}$, 如通过 测定感热和潜热通量或利用辐射平衡和其他相关变 量由 Penman-Monteith 公式或梯度原理重新计算得到 冠层阻抗 ${ }^{[14]}$.

现有研究多集中在以 Jarvis 气孔导度(气孔阻抗) 模型为基础, 考虑冠层光合有效辐射分布 ${ }^{[5,15]}$ 、阴阳 叶 ${ }^{[5,16,17]}$ 以及叶面积 ${ }^{[18,19]}$ 等实现冠层阻抗的尺度提升, matal conductance. Chin Sci Bull, 2013, 58, doi: 10.1007/s11434-013-5858-3 
或以 Leuning-Ball 气孔导度(气孔阻抗)模型为基础, 建立叶片尺度或冠层尺度单一尺度光合-蒸腾-气孔 导度耦合模型 ${ }^{[20 ~ 22]}$, 而建立叶片尺度 Leuning-Ball气 孔导度提升的冠层阻抗估算模型研究较少. 本文以 华北典型地区冬小麦为例, 率定 Leuning-Ball 和 Jarvis 气孔导度模型的参数, 以光合有效辐射作为尺 度转换因子, 构建 Leuning-Ball 和 Jarvis 的冠层阻抗 尺度提升模型，对比和验证两种尺度提升模型的应 用效果，篮选适合华北典型地区的冠层阻抗基于叶 片气孔导度的提升方法.

\section{1 材料与方法}

\section{1 试验区概况}

华北地区为我国的主要粮食产区之一，典型试 验区中国水利水电科学研究院大兴节水灌溉试验研 究基地(北纬 $39^{\circ} 37^{\prime}$, 东经 $116^{\circ} 26^{\prime}$ )主要是冬小麦-夏 玉米轮作制度种植. 田间观测试验于 2007 2008 年和 2008 2009 年冬小麦主要生长季进行. 试验区为半干 旱大陆性季风气候, 年平均气温为 $12.1^{\circ} \mathrm{C}$, 全年大于 $10^{\circ} \mathrm{C}$ 的有效积温为 $4730^{\circ} \mathrm{C}$, 无霜期平均为 $185 \mathrm{~d}$, 全年 日照时数约为 2600 h. 0 100 cm 深度为砂壤土, 平均 田间持水率和土壤容重分别为 $30.58 \%$ 和 $1.58 \mathrm{~g} \mathrm{~cm}^{-3}$.

\section{2 试验观测}

(i ) 蒸散量 $E T$. 采用浴度相关仪(美国 Campbell Scientific 公司)测定, 其计算公式为

$$
\lambda E T=\lambda \rho_{a} \overline{w^{\prime} q^{\prime}},
$$

式中, $E T$ 为蒸散量, $\mathrm{mm} \mathrm{s}^{-1} ; \lambda$ 为水的汽化潜热, $\mathrm{J} \mathrm{kg}^{-1}$; $\rho_{a}$ 为空气密度, $\mathrm{kg} \mathrm{m}^{-3} ; w^{\prime}$ 为垂直风速脉动量, $\mathrm{m} \mathrm{s}^{-1} ; q^{\prime}$ 为水汽密度脉动值, $\mathrm{g} \mathrm{m}^{-3}$.

本研究采用的浴度相关仪观测冬小麦生长季的 温度、湿度、三维风速的脉动值以及土壤热通量. 仪 器田间安装高度为 $3.1 \mathrm{~m}$, 采样频率是 $10 \mathrm{~Hz}$, 统计平 均时间是 $30 \mathrm{~min}, \mathrm{CO}_{2}$ 通量和潜热通量实时进行 WPL(空气密度脉动)修正, 显热通量进行超声虚温 (湿度影响)修正 ${ }^{[23]}$. 利用当地冬小麦田间观测数据, 对使用的浴度相关仪进行了能量闭合校核, 能量闭 合度达到 $80 \%$, 表明该设备工作性能良好 ${ }^{[24]}$. 在涡度 相关仪数据处理过程中, 依据以下原则对异常数据 进行剔除：(1) 降水日观测数据; (2) 明显超出物理 含义的数据; (3) 传感器状态出现异常的数据; (4)
冬小麦灌水日的数据. 此外, 通过计算日内波文比修 正潜热通量来消除能量不闭合引起的误差 ${ }^{[25]}$.

(ii) 叶片气孔导度、净光合速率. 在浴度相关 仪观测范围内, 随机选取均匀分布的 6 株典型冬小麦 作为代表植株, 采用 Li-6400 光合作用测定系统( LiCOR, USA), 在冬小麦生长旺盛季节, 选择晴朗无云 天气每 5 天测定叶片气孔导度 $\left(g_{\mathrm{s}}\right)$ 、净光合速率 $\left(P_{\mathrm{n}}\right)$ 、 光合有效辐射 $\left(\mathrm{PAR}_{\mathrm{a}}\right)$ 、温度和湿度, 选择大小均匀且 受光方向一致的 3 4 片旗叶从 8:00 16:00 每 2 小时 测定 1 次, 数据稳定后开始记录, 并分别取均值作为 该时刻冬小麦叶片 $g_{\mathrm{s}}, P_{\mathrm{n}}$ 和相关环境因子的测定结果.

（iii）株高和叶面积指数. 选取 10 株有代表性 的植株, 每 15 天测定其株高 $\left(h_{\mathrm{c}}\right)$ 和叶面积指数 $(\mathrm{LAI})$. 作物株高用钢卷尺测量, 叶面积用长 $\times$ 宽 $\times$ 修正系数 的方法计算, 根据种植密度得到整体叶面积指数, 修 正系数取值为 0.78 .

(iv) 消光系数. 采用 SunScan 冠层分析系统 (Dynamax, Inc., USA)测定消光系数, 在冬小麦生长 旺盛期, 每 15 天测定 1 次, 于 10:00 12:00 连续测定 冠层顶部和底部的光合有效辐射并取其平均值, 最 后根据实测的叶面积指数, 求得冠层消光系数.

\section{2 数学模型}

以光合有效辐射作为尺度转换因子, 以 LeuningBall 气孔导度模型为基础, 联立光响应修正模型, 光 合有效辐射观测衰减模型, 实现冠层阻抗的估算, 同 时建立以 Jarvis 气孔导度为基础的冠层阻抗估算 模型。

\section{1 叶片气孔导度估算模型}

( i ) Leuning-Ball 气孔导度模型. 由于相对湿 度和低 $\mathrm{CO}_{2}$ 浓度对 Ball 气孔导度模型的限制问题, Leuning 对 Ball 模型进行修正, 建立 Leuning-Ball 气 孔导度模型:

$$
g_{\mathrm{s}}=m \frac{P_{\mathrm{n}}}{\left(C_{\mathrm{s}}-\Gamma\right)\left(1+\mathrm{VPD}_{\mathrm{s}} / \mathrm{VPD}_{0}\right)}+g_{\mathrm{s} 0},
$$

式中, $g_{\mathrm{s}}$ 是气孔导度, $\mathrm{mol} \mathrm{m} \mathrm{m}^{-2} \mathrm{~s}^{-1} ; P_{\mathrm{n}}$ 是净光合速率, $\mu \mathrm{mol} \mathrm{m}{ }^{-2} \mathrm{~s}^{-1} ; \Gamma$ 是 $\mathrm{CO}_{2}$ 补偿点, $\mu \mathrm{mol} \mathrm{mol}{ }^{-1} ; C_{\mathrm{s}}$ 为叶表 面 $\mathrm{CO}_{2}$ 浓度, $\mu \mathrm{mol} \mathrm{mol}{ }^{-1} ; \mathrm{VPD}_{\mathrm{s}}$ 是叶面的水汽压差, $\mathrm{kPa} ; m$ 和 $\mathrm{VPD}_{0}$ 为经验常数, $g_{\mathrm{s} 0}$ 是在光补偿点处的 $g_{\mathrm{s}}$ 值，相关计算公式见文献[26]. 
(ii) Jarvis 气孔导度模型. Jarvis 认为气孔导度 是多个环境因子综合作用的产物, 可通过气孔导度 对单一环境因子反应叠加得到多个环境因子同时变 化时对叶片气孔导度的综合影响, Yu 等人 ${ }^{[27]}$ 认为对 于短时期而言, 选择双因子 Jarvis 模型, 其精度已经 满足要求. 因此, 参考张宝忠等人 ${ }^{[28]}$ 建立的双因子 Jarvis 模型, 基于光合有效辐射 $\mathrm{PAR}_{\mathrm{a}}$ 和饱和水汽压 差 VPD 构建冬小麦叶片气孔导度 $g_{\text {s }}$ 估算模型:

$g_{\mathrm{s}}=g_{\mathrm{s}}\left(\mathrm{PAR}_{\mathrm{a}}\right) f(\mathrm{VPD})=\frac{\mathrm{PAR}_{\mathrm{a}}}{\mathrm{PAR}_{\mathrm{a}}+\alpha} \exp (-\beta \cdot \mathrm{VPD})$,

式中, $\mathrm{PAR}_{\mathrm{a}}$ 为叶面截获的光合有效辐射, $\mu \mathrm{mol} \mathrm{m} \mathrm{m}^{-2} \mathrm{~s}^{-1}$; $\mathrm{VPD}$ 是饱和水汽压差, $\mathrm{kPa} ; \alpha, \beta$ 为经验系数.

\section{2 光响应修正模型}

考虑直角双曲线模型以及非直角双曲线模型光 响应模型无法较好地描述光抑制现象及给出的光饱 和点小于实测值, $\mathrm{Ye}$ 等人 ${ }^{[29}$ 提出了光响应修正模型:

$$
P_{n}=a \times \frac{1-c \times \mathrm{PAR}_{\mathrm{a}}}{1+b \times \mathrm{PAR}_{\mathrm{a}}} \times \mathrm{PAR}_{\mathrm{a}}-R_{\mathrm{d}},
$$

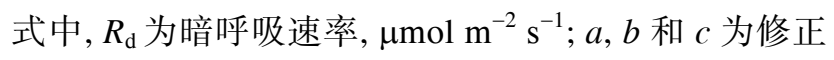
系数, 其余变量含义同上.

\section{3 光合有效辐射冠层衰减模型}

光在作物冠层衰减规律服从 Beer-Lambert 定律, 因此, 叶片截获的光合有效辐射 $\mathrm{PAR}_{\mathrm{a}}$ 由式(5), (6)计 算:

$$
\begin{gathered}
\mathrm{PAR}=\mathrm{PAR}_{\mathrm{h}} \exp (-K \xi), \\
\mathrm{PAR}_{\mathrm{a}}=-\mathrm{dPAR} / \mathrm{d} \xi=K \cdot \mathrm{PAR}_{\mathrm{h}} \cdot \exp (-K \xi),
\end{gathered}
$$

式中, PAR, $\mathrm{PAR}_{\mathrm{h}}$ 为冠层某一高度以及冠层顶部的光 合有效辐射, $\mu \mathrm{mol} \mathrm{m} \mathrm{m}^{-2} \mathrm{~s}^{-1} ; \xi$ 为冠层某一高度到冠层 顶的叶面积指数; $K$ 为消光系数.

\section{4 冠层阻抗估算模型}

以光合有效辐射作为尺度转换因子 ${ }^{[5,30]}$, 假定下 垫面均匀分布且忽略土壤蒸发影响、冠层内水汽压变 化以及 $\mathrm{CO}_{2}$ 浓度变化状况下 ${ }^{[31,32]}$, 对式(3)进行积分, 获得基于冬小麦叶片气孔导度提升的冠层阻抗 $\left(r_{\mathrm{c}}\right)$ 的 估算模型：

$$
1 / r_{\mathrm{c}}=\int g_{\mathrm{s}} \mathrm{d} \xi=\int_{0}^{\mathrm{LAI}} g_{\mathrm{s}} \mathrm{d} \xi .
$$

将式(2), (4)和(6)代人式(7), 则 Leuning-Ball 尺度提
升模型为

$$
\begin{aligned}
& 1 / r_{\mathrm{c}}=\left(g_{\mathrm{sw}}-R_{\mathrm{d}}\right) \times \mathrm{LAI}+\frac{m \times a \times\left(k \times c \times \mathrm{PAR}_{\mathrm{h}}-1\right)}{K \times b \times\left(C_{\mathrm{s}}-\Gamma\right)\left(1+\mathrm{VPD}_{\mathrm{s}} / \mathrm{VPD}_{0}\right)} \\
& \times \ln \left(1+K \times b \times \mathrm{PAR}_{\mathrm{h}} \times \exp (-K \times \mathrm{LAI}) / 1+K \times b \times \mathrm{PAR}_{\mathrm{h}}\right) .
\end{aligned}
$$

将式(3)和(6)代人式(7), 则 Jarvis 尺度提升模型为

$$
\begin{aligned}
1 / r_{\mathrm{c}}= & \frac{\exp (-\beta \times \mathrm{VPD})}{K} \\
& \times \ln \left(\frac{K \times \mathrm{PAR}_{\mathrm{h}}+\alpha}{K \times \mathrm{PAR}_{\mathrm{h}} \times \exp (-K \times \mathrm{LAI})+\beta}\right) .
\end{aligned}
$$

\section{5 冠层阻抗}

冠层阻抗 $\left(r_{\mathrm{c}}\right)$ 通过 Penman-Monteith 模型推算:

$$
\begin{gathered}
r_{\mathrm{c}}=\frac{r_{\mathrm{a}}\left(\Delta\left(R_{\mathrm{n}}-G\right)+\rho_{\mathrm{a}} C_{\mathrm{p}} \operatorname{VPD} / r_{\mathrm{a}}-\lambda E T(\Delta+\gamma)\right)}{\gamma \lambda E T}, \\
r_{\mathrm{a}}=\frac{\ln \left(\frac{z-d}{h_{\mathrm{c}}-d}\right) \ln \left(\frac{z-d}{z_{0}}\right)}{k^{2} u_{\mathrm{z}}} .
\end{gathered}
$$

零平面位移高度 $(d)$ 和下垫面粗踈长度 $\left(z_{0}\right)$ 随作物 高度 $\left(h_{\mathrm{c}}\right)$ 的改变而变化, 其表达式为 ${ }^{[33]}$

$$
\begin{aligned}
& d=0.63 h_{\mathrm{c}}, \\
& z_{0}=0.13 h_{\mathrm{c}},
\end{aligned}
$$

式中, $r_{\mathrm{a}}$ 为空气动力学阻抗, $\mathrm{s} \mathrm{m}^{-1} ; r_{\mathrm{c}}$ 为冠层阻抗, $\mathrm{s} \mathrm{m}^{-1} ; R_{\mathrm{n}}$ 为净辐射, $\mathrm{W} \mathrm{m}^{-2} ; G$ 为土壤热通量, $\mathrm{W} \mathrm{m}^{-2} ; \gamma$ 为湿度计常数, $\mathrm{kPa}{ }^{\circ} \mathrm{C}^{-1} ; \Delta$ 为饱和水汽压-温度曲线 的斜率, $\mathrm{kPa}{ }^{\circ} \mathrm{C}^{-1} ; C_{\mathrm{p}}$ 为空气的定压比热, $\mathrm{J} \mathrm{kg}^{-1} \mathrm{~K}^{-1} ; k$ 为 Karman 常数, 取值为 $0.41 ; z$ 为参考高度, 取值为 $2 \mathrm{~m} ; u_{\mathrm{z}}$ 为参考高度处的风速, $\mathrm{m} \mathrm{s}^{-1} ; h_{\mathrm{c}}$ 为冠层高度, $\mathrm{m}$; 其余变量含义同上.

\section{6 模型评价指标}

模型模拟效果主要采用确定系数 $\left(R^{2}\right)$, 均方根误 差 (RMSE), 平均绝对误差 (AAE), 平均相对误差 (ARE) 以及模型有效性指数 $(E F)$ 进行模型评价, 其计 算公式详见参考文献[34].

\section{3 结果与讨论}

\section{1 叶片气孔阻力和冠层阻抗日变化的差异性}

冬小麦叶片气孔阻力 $\left(r_{\mathrm{L}}\right)$ 和冠层阻抗 $\left(r_{\mathrm{c}}\right)$ 的日变 
化过程如图 1 所示, 2007 2008 年和 2008 2009 年冬 小麦叶片气孔阻力 $\left(r_{\mathrm{L}}\right)$ 和冠层阻抗 $\left(r_{\mathrm{c}}\right)$ 的日变化过程 在不同的生育阶段(叶龄)以及不同的气象条件下呈 现较一致的变化, 两个生长季冬小麦叶片气孔阻力 $\left(r_{\mathrm{L}}\right)$ 高于冠层阻抗 $\left(r_{\mathrm{c}}\right)$, 增幅分别为 $0.85 \% \sim 487.46 \%$ 和 $-24.54 \%$ 223.26\%. 采用非参数检验的 Friedman $(\mathrm{F})$ 检验类型对冬小麦叶片气孔阻力和冠层阻抗的日变 化进行差异性分析(图 1), 两个生长季的冬小麦典型 日叶片气孔阻力和冠层阻抗在 $a=0.05$ 水平上显著差 异的组数为 4 组. 因此, 叶片以及冠层尺度水汽传输 阻力存在显著的尺度差异, 叶片尺度的 $r_{\mathrm{L}}$ 不能代替 冠层尺度 $r_{\mathrm{c}}$, 需要进行尺度转换研究.

2008 年 5 月 20 日 14 时叶片气孔阻力值较大(图 $1(b))$, 该时刻测量重复值分别为 $378.76,458.22$ 以及 $1134.38 \mathrm{~s} \mathrm{~m}^{-1}$, 同期风速分别是 $0.45,0.43$ 和 $0.13 \mathrm{~m} \mathrm{~s}^{-1}$. 徐惠风等人 ${ }^{[35]}$ 认为气孔阻力与风速呈显著负相关关 系, 因此风速的降低致使 14 时气孔阻力均值变大. 同时刻冠层阻抗与其余时刻的数值差别较小, 冠层 阻抗的计算是基于浴度相关仪 $30 \mathrm{~min}$ 统计平均时间 进行反推. 表明从叶片尺度到冠层尺度空间尺度转 换的同时, 带来响应的时间尺度转换问题, 导致时空 尺度转换的同时进行 ${ }^{[36]}$.

\section{2 叶片气孔导度模型的率定与验证}

(i) 叶片气孔导度模型的率定. 利用 2007 2008 年冬小麦生育期实测的数据 $(n=990)$ 对 Leuning-Ball 和 Jarvis 叶片气孔导度模型进行参数率定, 得到 LeuningBall 模型参数 $m=15.293, \mathrm{VPD}_{0}=0.657 \mathrm{kPa}$ 和 $g_{\mathrm{s} 0}=0.123$, Jarvis 模型参数 $\alpha=265.668$ 和 $\beta=0.4$. 由叶片气孔导度 模拟值与实测值回归关系(图 2)和不同气孔导度模型 评价统计指标(表 1)可知, Leuning-Ball 模型和 Jarvis 模型实测值和模拟值间相关方程的斜率分别为 0.95 和 0.93 , 确定系数 $\left(R^{2}\right)$ 为 0.65 和 0.47 , 均方根误差 (RMSE) 为 0.08 和 $0.09 \mathrm{~mol} \mathrm{~m}^{-2} \mathrm{~s}^{-1}$, 平均绝对误差 (AAE) 为 0.06 和 $0.07 \mathrm{~mol} \mathrm{~m}^{-2} \mathrm{~s}^{-1}$, 模型有效性指数 $(E F)$ 为 0.71 和 0.61 . 因此, Leuning-Ball 模型和 Jarvis 模型能够有效模拟冬小麦叶片气孔导度对环境因子 的响应.

(ii) 叶片气孔导度估算模型的验证. 利用 2008 2009 年冬小麦生育期实测的数据 $(n=1440)$ 对 LeuningBall 和 Jarvis 叶片气孔导度估算模型进行验证, 由叶 片气孔导度验证值与实测值回归关系(图 3)和不同气 孔导度模型评价统计指标(表 1)可知, Leuning-Ball 模 型和 Jarvis 模型实测值和模拟值间相关方程的斜率分 别为 1.06 和 0.84 , 确定系数 $\left(R^{2}\right)$ 为 0.67 和 0.18 , 均方
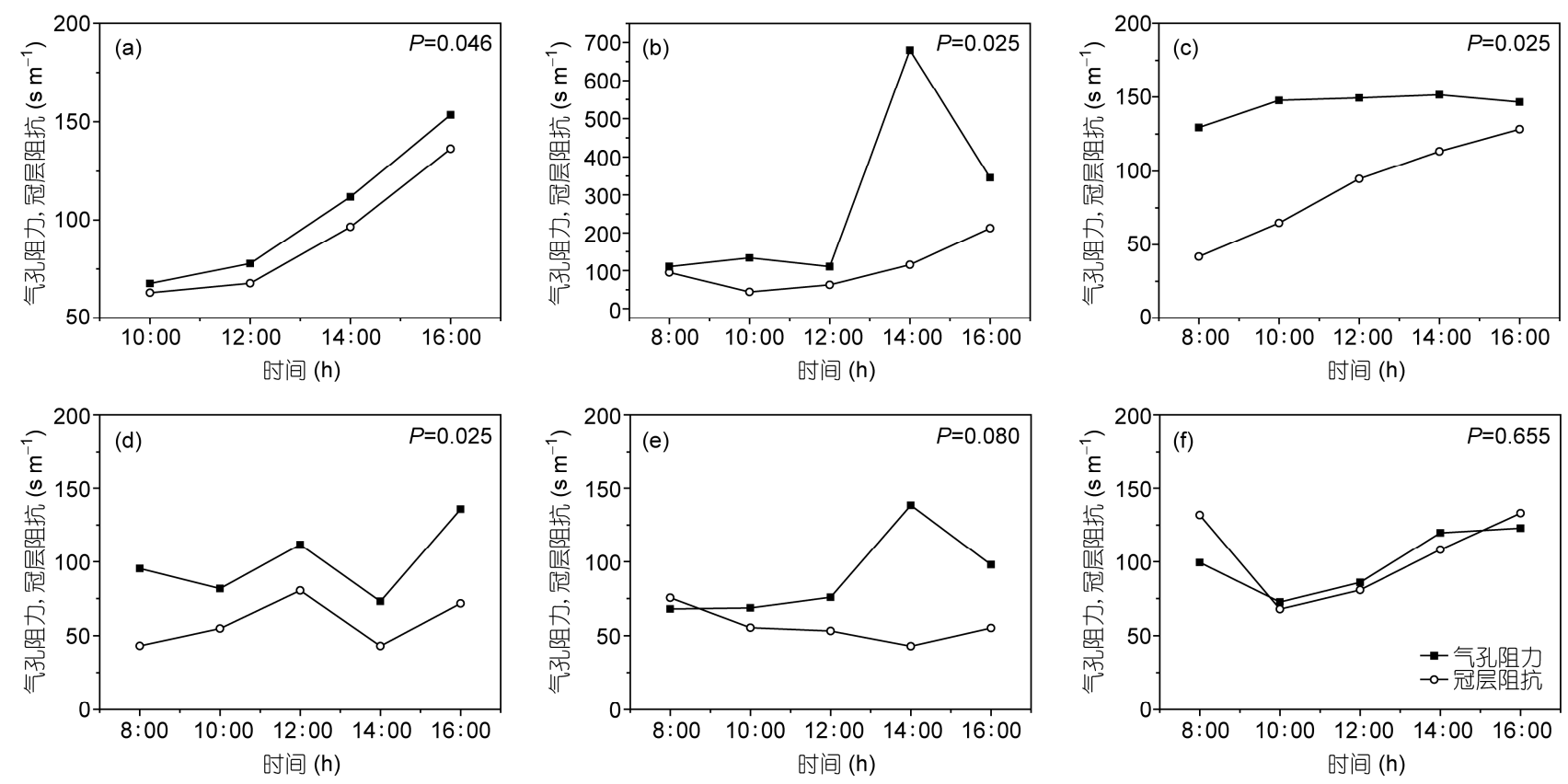

图 1 典型日冬小麦叶片气孔阻力和冠层阻抗日变化曲线

(a) 2008-05-05; (b) 2008-05-20; (c) 2008-06-11; (d) 2009-05-02; (e) 2009-05-08; (f) 2009-05-30 

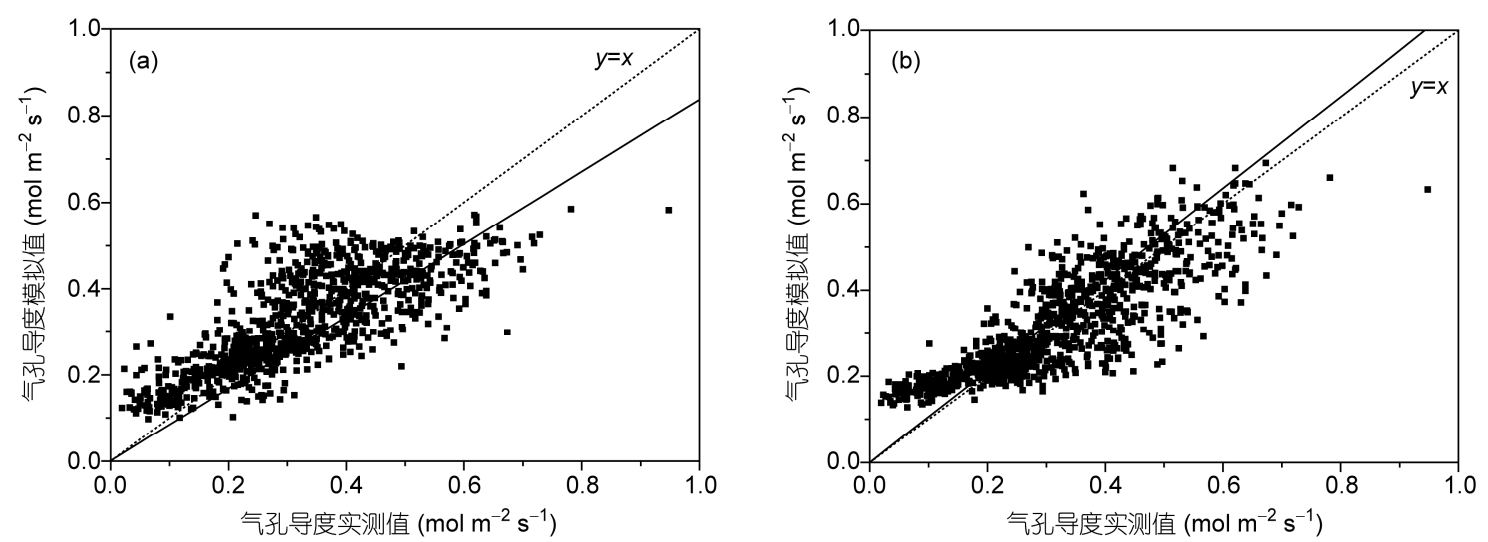

图 22007 2008 年冬小麦生长期叶片气孔导度实测值与模拟值关系图 (a) Jarvis 气孔导度估算模型; (b) Leuning-Ball 气孔导度估算模型
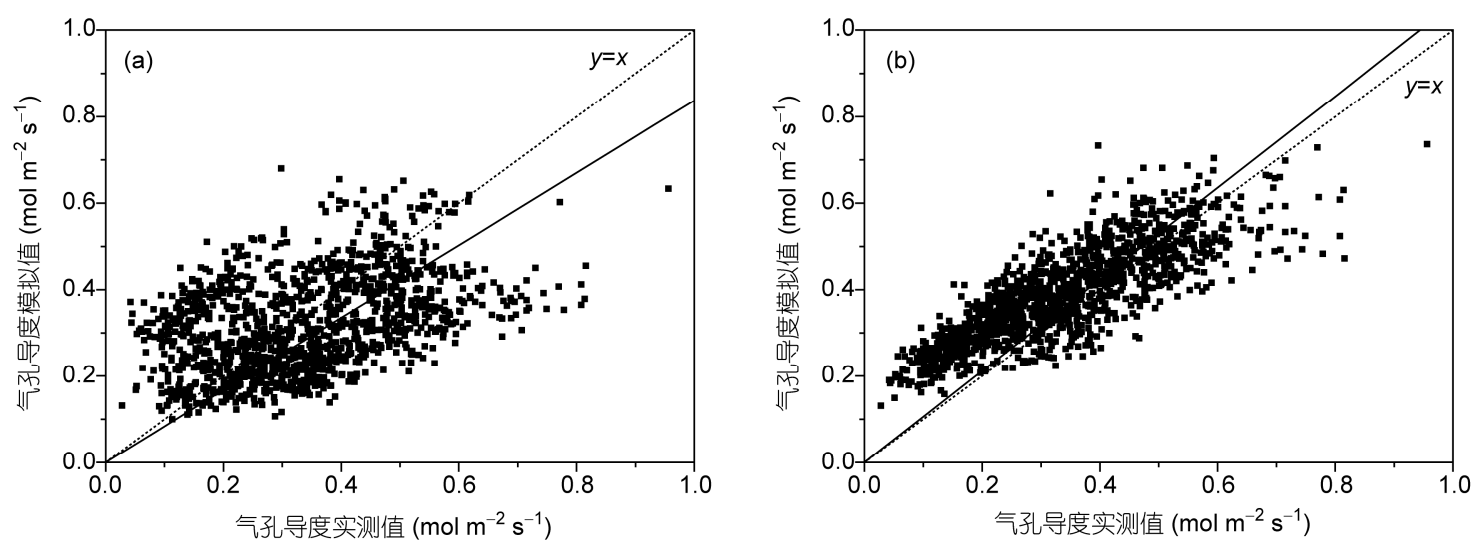

图 $32008 \sim 2009$ 年冬小麦生长期叶片气孔导度实测值与模拟值关系图 (a) Jarvis 气孔导度估算模型; (b) Leuning-Ball 气孔导度估算模型

表 1 两个冬小麦生长季叶片气孔导度模拟值与实测值的统计分析

\begin{tabular}{|c|c|c|c|c|c|c|c|}
\hline & & $b$ & $R^{2}$ & $\begin{array}{c}\text { RMSE } \\
\left(\mathrm{mol} \mathrm{m}^{-2} \mathrm{~s}^{-1}\right)\end{array}$ & $\begin{array}{c}\mathrm{AAE} \\
\left(\mathrm{mol} \mathrm{m}^{-2} \mathrm{~s}^{-1}\right)\end{array}$ & $\operatorname{ARE}(\%)$ & $E F$ \\
\hline \multirow{2}{*}{$2007 \sim 2008$ 年 } & Leuning-Ball 模型 & 0.95 & 0.65 & 0.08 & 0.06 & 2.03 & 0.71 \\
\hline & Jarvis 模型 & 0.93 & 0.47 & 0.09 & 0.07 & 3.46 & 0.61 \\
\hline \multirow{2}{*}{$2008 \sim 2009$ 年 } & Leuning-Ball 模型 & 1.06 & 0.67 & 0.10 & 0.09 & 1.53 & 0.55 \\
\hline & Jarvis 模型 & 0.84 & 0.18 & 0.15 & 0.12 & 2.69 & 0.07 \\
\hline
\end{tabular}

根误差(RMSE)为 0.10 和 $0.15 \mathrm{~mol} \mathrm{~m}^{-2} \mathrm{~s}^{-1}$, 平均绝对 误差(AAE)为 0.09 和 $0.12 \mathrm{~mol} \mathrm{~m}^{-2} \mathrm{~s}^{-1}$, 模型有效性指 数 $(E F)$ 为 0.55 和 0.07 . Leuning-Ball 模型能够较 Jarvis 模型更好地解释华北地区冬小麦叶片气孔导度对环 境因子的响应变化, Jarvis 双因子(光合有效辐射和饱 和水汽压)模型不能反映 2008 2009 年冬小麦叶片气 孔导度的变化特点, 其模型参数具有一定的年际限
制, 这点与 $\mathrm{Yu}$ 等人 ${ }^{[37]}$ 研究认为 2 变量模型的估算精 度可满足短时间尺度要求，而对于长时间尺度，3 4 变量模型的估算精度较低类似.

\section{3 冠层阻抗估算模型验证}

结合不同时期冬小麦消光系数和叶面积指数(表 2), 构建的 Leuning-Ball 和 Jarvis 尺度提升模型, 利 
表 2 不同时期冬小麦消光系数和叶面积指数

\begin{tabular}{ccc}
\hline 日期 & 消光系数 & 叶面积指数 \\
\hline $2008-05-05$ & 0.69 & 5.01 \\
$2008-05-20$ & 0.83 & 6.21 \\
$2008-06-11$ & 0.78 & 4.83 \\
$2009-05-02$ & 0.56 & 4.68 \\
$2009-05-08$ & 0.69 & 5.73 \\
$2009-05-30$ & 0.58 & 5.12 \\
\hline
\end{tabular}

用 2007 2008 年和 2008 2009 年冬小麦生育期冠层阻 抗实测值对模型进行验证，由冬小麦生长期典型时 刻冠层阻抗实测值与模拟值变化过程(图 4)可知, 基 于 Leuning-Ball气孔导度模型构建的冠层阻抗估算模 型的模拟值与实测值的日变化趋势基本一致. 由不 同冠层阻抗估算模型评价统计指标(表 3)可知, 模拟 值与实测值的相关方程的斜率分别为 1.01 和 1.12 , 确定系数为 0.77 和 0.46 , 均方根误差为 23.37 和 31.73 $\mathrm{s} \mathrm{m}^{-1}$, 平均绝对误差为 17.84 和 $23.38 \mathrm{~s} \mathrm{~m}^{-1}$, 相对误
差为 $19.67 \%$ 和 32.10\%, 这表明基于 Leuning-Ball 气 孔导度模型构建的冠层阻抗估算模型(简称 LeuningBall 尺度提升模型)较为适合当地的应用, 但在 $r_{\mathrm{c}}$ 实 测值较大时，两者的误差增加，这也说明以 LeuningBall 气孔导度模型为基础, 以 PAR 作为尺度转换因 子的尺度提升模型只能阐述麦田冠层内水汽传输阻 力, 未考虑土壤水分、土壤蒸发的影响, 而 PenmanMonteith 方程反推 $r_{\mathrm{c}}$ 值的过程中包含地表阻力的影 响. 因此, Leuning-Ball 气孔导度模型应耦合土壤水 分因子, 考虑水分胁迫对叶片气孔导度的影响, 如 Egea 等人 ${ }^{[38]}$ 在式(2)方程右边第一项引人水分胁迫因 子 $\beta^{\prime}$, 改进了净光合速率-气孔导度耦合模型.

基于 Jarvis气孔导度模型构建的冠层阻抗估算模 型(简称 Jarvis 尺度提升模型)的模拟值与实测值的变 化趋势基本一致，但差别显著，两者间相关方程的斜 率为 0.62 和 1.76 , 确定系数为 0.63 和 0.34 , 均方根误 差为 67.38 和 $97.41 \mathrm{~s} \mathrm{~m}^{-1}$, 平均绝对误差为 49.33 和 $64.92 \mathrm{~s} \mathrm{~m}^{-1}$ 、平均相对误差为 $50.69 \%$ 和 $76.14 \%$. 表明
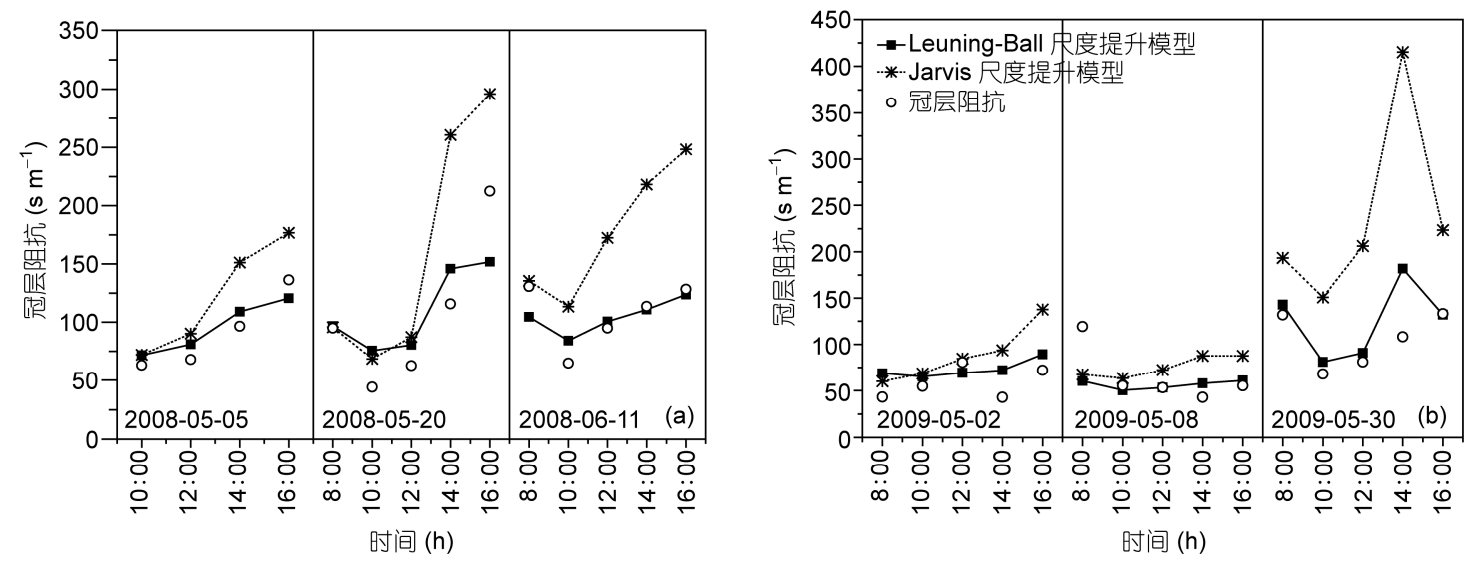

图 4 两个生长季冬小麦典型时刻冠层阻抗实测值与模拟值变化过程

(a) 2007 2008 年; (b) 2008 2009 年

表 3 两个冬小麦生长季冠层阻抗模拟值与实测值的统计分析 ${ }^{\text {a) }}$

\begin{tabular}{|c|c|c|c|c|c|c|c|}
\hline & & 相关方程 & $R^{2}$ & $\begin{array}{l}\text { RMSE } \\
\left(\mathrm{s} \mathrm{m}^{-1}\right)\end{array}$ & $\begin{array}{c}\mathrm{AAE} \\
\left(\mathrm{s} \mathrm{m}^{-1}\right)\end{array}$ & $\begin{array}{c}\text { ARE } \\
(\%)\end{array}$ & $E F$ \\
\hline \multirow[t]{2}{*}{ 2007 2008 } & $\begin{array}{l}\text { Leuning-Ball 尺度 } \\
\text { 提升模型 }\end{array}$ & $r_{\mathrm{co}}=1.01 r_{\mathrm{cp}}$ & 0.78 & 23.37 & 17.84 & 19.67 & 0.66 \\
\hline & Jarvis 尺度提升模型 & $r_{\mathrm{co}}=0.62 r_{\mathrm{cp}}$ & 0.63 & 67.38 & 50.69 & 49.33 & 0.42 \\
\hline \multirow[t]{2}{*}{$2008 \sim 2009$} & $\begin{array}{l}\text { Leuning-Ball 尺度 } \\
\text { 提升模型 }\end{array}$ & $r_{\mathrm{co}}=1.12 r_{\mathrm{cp}}$ & 0.46 & 31.73 & 23.38 & 32.10 & 0.57 \\
\hline & Jarvis 尺度提升模型 & $r_{\mathrm{co}}=1.76 r_{\mathrm{cp}}$ & 0.34 & 97.41 & 64.92 & 76.14 & 0.22 \\
\hline
\end{tabular}

a) $r_{\mathrm{co}}$ 为利用 $\mathrm{P}-\mathrm{M}$ 方程反推的冠层阻抗; $r_{\mathrm{cp}}$ 为基于叶片气孔导度尺度转换提升的冠层阻抗 
应用以光合有效辐射 $\left(\mathrm{PAR}_{\mathrm{a}}\right)$ 和饱和水汽压差 $(\mathrm{VPD})$ 为 主要影响因子的 Jarvis气孔导度模型估算冬小麦冠层 阻抗具有一定的困难, 这与 Rochette 等人建立的阴阳 叶模型 ${ }^{[5,39,40]}$ 能够有效解释作物冠层导度有一定差别, 原因在于阴阳叶模型区分了自然状态下作物阴阳叶 的气孔导度、光合有效辐射以及叶面积指数, 这能够 提高冠层导度(阻抗)的模拟精度.

\section{4 敏感性分析}

当 Leuning-Ball 尺度提升模型和 Jarvis 尺度提升 模型输人项分别变化 $\pm 10 \%$ 时, 冠层阻抗模拟值相对 误差(ARE)变化范围如表 4 所示. 对比两个模型可知, Jarvis 模型对辐射以及叶面积指数两输人项的敏感性 要高于 Leuning-Ball 模型. 因此, 区分阴阳叶可以更 有效地提高 Jarvis 经验模型的精度.

当 Leuning-Ball 尺度提升模型和 Jarvis 尺度提升 模型参数分别变化 $\pm 10 \%$ 时, 冠层阻抗模拟值相对误 差(ARE)变化范围如表 4 所示. 其中以 Leuning-Ball 气孔导度模型参数 $\left(m\right.$ 和 $g_{\mathrm{s} 0}$ ), 光响应修正模型参数 $(a)$ 以及 $\mathrm{CO}_{2}$ 补偿点 $(\Gamma)$ 对 Leuning-Ball 尺度提升模型的 影响程度较大, 光响应修正模型参数 ( $c$ 和 $R_{\mathrm{d}}$ ) 次之; 叶片尺度 Leuning-Ball 模型参数 $\left(m, \mathrm{VPD}_{0}\right.$ 和 $\left.g_{\mathrm{s} 0}\right)$ 和 Jarvis 气孔导度模型参数 $(\alpha$ 和 $\beta$ )在 $-10 \% \sim 10 \%$ 变化时, 冠层阻抗模拟值相对误差变化范围为-9.06\% $8.22 \%$
和 $-5.84 \% \sim 6.13 \%$, 因此, Jarvis 模型的参数 $(\alpha$ 和 $\beta)$ 在 冠层尺度上的敏感性较小.

\section{4 结论}

通过 2007 2008 年和 2008 2009年两个生长季冬 小麦田间试验研究, 揭示了叶片和冠层尺度冬小麦 水汽传输阻抗的差异性, 率定了 Leuning-Ball 和 Jarvis 气孔导度模型的参数, 得到了冠层阻抗的 LeuningBall 和 Jarvis 尺度提升模型, 验证和对比了两种尺度 提升模型的应用效果,篮选了适合华北典型地区冠 层阻抗基于叶片气孔导度的提升方法.

两个生长季的冬小麦叶片气孔阻抗整体高于冠 层阻抗，幅度为 $0.85 \%$ 487.46\%和-24.54\% 223.26\%, 利用非参数检验的 Friedman 检验类型进行检验, 叶 片、冠层尺度水汽传输阻力存在显著的尺度差异;

两个生长季的冬小麦叶片 Leuning-Ball气孔导度 模型具有较 Jarvis气孔导度模型更好地解释田间冬小 麦叶片气孔导度的能力, Jarvis 气孔导度模型对长时 期气孔导度的估算精度比较低. 以光合有效辐射作 为尺度转换因子, Leuning-Ball 尺度提升模型具有较 Jarvis 尺度提升模型更有效地估算冠层阻抗变化的能 力, Jarvis 尺度提升模型参数在冠层尺度上的敏感性 较小. 基于 Leuning-Ball 气孔导度尺度提升模型可应 用华北典型地区冬小麦水汽传输阻抗尺度提升研究.

表 4 叶片气孔导度提升的冠层阻抗模型输入项和参数敏感性分析

\begin{tabular}{|c|c|c|c|c|c|c|}
\hline & \multirow{2}{*}{ 输人项 } & \multicolumn{2}{|c|}{ 相对误差(\%) } & \multirow{2}{*}{ 模型参数 } & \multicolumn{2}{|c|}{ 相对误差(\%) } \\
\hline & & 10 & -10 & & 10 & -10 \\
\hline \multirow{8}{*}{ Leuning-Ball 尺度提升模型 } & $K$ & $-2.16 \sim-0.37$ & $0.66 \sim 2.57$ & $m$ & $-6.81 \sim-2.35$ & $2.46 \sim 7.14$ \\
\hline & LAI & $-8.59 \sim-5.44$ & $8.03 \sim 11.44$ & $\mathrm{VPD}_{0}$ & $-4.66 \sim-1.72$ & $1.88 \sim 5.40$ \\
\hline & $\mathrm{VPD}_{\mathrm{s}}$ & $-3.07 \sim 4.87$ & $-11.26 \sim-2.66$ & $\mathrm{~g}_{\mathrm{s} 0}$ & $-9.06 \sim-2.62$ & $2.77 \sim 8.22$ \\
\hline & $\mathrm{PAR}_{\mathrm{h}}$ & $-9.61 \sim-1.70$ & $-3.98 \sim 4.09$ & $a$ & $-7.26 \sim-3.01$ & $3.20 \sim 8.49$ \\
\hline & $C_{s}$ & $3.34 \sim 8.82$ & $-8.63 \sim-1.79$ & $b$ & $0.57 \sim 4.73$ & $-4.93 \sim-0.59$ \\
\hline & & & & $c$ & $3.63 \sim 7.70$ & $-6.67 \sim-3.38$ \\
\hline & & & & $R_{d}$ & $-1.84 \sim 2.35$ & $-2.09 \sim 5.16$ \\
\hline & & & & $\Gamma$ & $-8.0 \sim-0.65$ & $-5.83 \sim 1.43$ \\
\hline \multirow{4}{*}{ Jarvis 尺度提升模型 } & $K$ & $-14.2 \sim-0.58$ & $-9.04 \sim 5.13$ & $\alpha$ & $2.97 \sim 5.11$ & $-4.10 \sim-2.97$ \\
\hline & LAI & $-16.51 \sim-5.20$ & $-5.98 \sim 10.16$ & $\beta$ & $2.67 \sim 6.13$ & $-5.84 \sim-2.60$ \\
\hline & VPD & $-1.25 \sim 14.13$ & $-21.29 \sim-2.60$ & & & \\
\hline & $\mathrm{PAR}_{\mathrm{h}}$ & $-17.57 \sim-0.75$ & $-6.04 \sim 7.02$ & & & \\
\hline
\end{tabular}




\section{参考文献}

1 Rana G, Katerji N, Mastrorilli M, et al. Validation of a model of actual evapotranspiration for water stressed soybeans. Agr Forest Meteorol, 1997, 86: 215-224

2 Shuttleworth W J, Wallace J S. Evaporation from sparse crops-An energy combination theory. Q J Roy Meteor Soc, 1985, 111: 839-855

3 Brenner A J, Incoll L D. The effect of clumping and stomatal response on evaporation from sparsely vegetated shrublands. Agr Forest Meteorol, 1997, 84: 187-205

4 Shuttleworth W J. Towards one-step estimation of crop water requirements. T Asabe, 2006, 49: 925-935

5 Irmak S, Mutiibwa D, Irmak A, et al. On the scaling up leaf stomatal resistance to canopy resistance using photosynthetic photon flux density. Agr Forest Meteorol, 2008, 148: 1034-1044

6 Kato T, Kimura R, Kamichika M. Estimation of evapotranspiration, transpiration ratio and water-use efficiency from a sparse canopy using a compartment model. Agr Water Manage, 2004, 65: 173-191

7 Rana G, Katerji N. Measurement and estimation of actual evapotranspiration in the field under mediterranean climate: A review. Eur J Agron, 2000, 13: 125-153

8 Anadranistakis M, Liakatas A, Kerkides P, et al. Crop water requirements model tested for crops grown in Greece. Agr Water Manage, 2000, 45: 297-316

9 Furon A, Warland J S, Wagner-Riddle C. Analysis of scaling-up resistances from leaf to canopy using numerical simulations. Agron J, 2007, 99: 1483-1491

10 Avissar R, Pielke R A. The impact of plant stomatal control on mesoscale atmospheric circulations. Agr Forest Meteorol, 1991, 54: 353-372

11 Yu G R, Nakayama K, Matsuoka N, et al. A combination model for estimating stomatal conductance of maize (Zea mays L.) Leaves over a long term. Agr Forest Meteorol, 1998, 92: 9-28

12 Beven K. A sensitivity analysis of the penman-monteith actual evapotranspiration estimates. J Hydrol, 1979, 44: 169-190

13 Black T A. Estimation of Areal Evapotranspiration. Budapest: IAHS Press, 1989

14 Oltchev A, Ibrom A, Constantin J, et al. Stomatal and surface conductance of a spruce forest: Model simulation and field measurements. Phys Chem Earth, 1998, 23: 453-458

15 Raupach M R. Vegetation-atmosphere interaction and surface conductance at leaf, canopy and regional scales. Agr Forest Meteorol, 1995, 73: $151-179$

16 申双和，孙照渤，陈镜明，等. 北方黑云杉冠内空气 CO2 浓度及其上方通量模拟。气象学报, 2005, 63: 6-17

17 Whitehead D, Okali D, Fasehun F E. Stomatal response to environmental variables in two tropical forest species during the dry season in Nigeria. J Appl Ecol, 1981, 18: 571-587

18 Zhang B Z, Kang S, Li F, et al. Comparison of three evapotranspiration models to bowen ratio-energy balance method for a vineyard in an arid desert region of northwest china. Agr Forest Meteorol, 2008, 148: 1629-1640

19 Zhou M C, Ishidaira H, Takeuchi K. Estimation of potential evapotranspiration over the yellow river basin: Reference crop evaporation or shuttleworth-wallace? Hydrol Process, 2007, 21: 1860-1874

20 Leuning R. A critical appraisal of a combined stomatal-photosynthesis model for C 3 plants. Plant Cell Environ, 1995, 18: 339-355

21 于强, 王天铎. 光合作用-蒸腾作用-气孔导度的耦合模型及 $\mathrm{C}_{3}$ 植物叶片对环境因子的生理响应. 植物学报, 1998, 40: 740-754

22 Sellers P J, Randall D A, Collatz G J, et al. A revised land surface parameterization (SIB2) for atmospheric gcms. Part I: Model formulation. J Clim, 1996, 9: 676-705

23 Webb E K, Pearman G I, Leuning R. Correction of flux measurements for density effects due to heat and water vapour transfer. Q J Roy Meteor Soc, 1980, 106: 85-100

24 刘国水. 作物蒸散量测定与计算方法研究. 硕士学位论文. 保定：河北农业大学, 2008

25 郭家选，梅旭荣，卢志光，等. 测定农田蒸散的浴度相关技术．中国农业科学, 2004, 37: 1172-1176

26 Yu G R, Zhuang J, Yu Z L. An attempt to establish a synthetic model of photosynthesis-transpiration based on stomatal behavior for maize and soybean plants grown in field. J Plant Physiol, 2001, 158: 861-874

27 Yu G R, Nakayama K, Lu H Q. Modeling stomatal conductance in maize leaves with environmental variables. J Agr Meteorol, 1996, 52: $321-330$

28 张宝忠, 刘钰, 许迪，等. 基于夏玉米叶片气孔导度提升的冠层导度估算模型. 农业工程学报,2011,27: 80-86

29 Ye Z P. A new model for relationship between irradiance and the rate of photosynthesis in oryza sativa. Photosynthetica, 2007, 45: $637-640$ 
30 Meidner H, Mansfield T A. Physiology of Stomata. Maidenhead: McGraw Hill, 1968

31 Warland J S, Furon A C, Wagner-Riddle C. Analysis of scaling-up resistances from leaf to canopy using numerical simulations. Agron J, 2007, 99: 1483-1491

32 Kaufmann M R. Leaf conductance as a function of photosynthetic photon flux density and absolute humidity difference from leaf to air. Plant Physiol, 1982, 69: 1018-1022

33 于贵瑞, 孙晓敏. 陆地生态系统通量观测的原理与方法. 北京：高等教育出版社, 2006

34 Mccuen R H, Knight Z, Cutter A G. Evaluation of the nash-sutcliffe efficiency index. J Hydrol Eng, 2006, 11: 597-602

35 徐惠风, 刘兴土, 沙箓, 等. 遮荫条件下乌拉苔草叶片气孔阻力与脯氨酸、叶绿素含量的研究. 农业系统科学与综合研究, 2004, 20: $232-234$

36 许迪. 灌溉水文学尺度转换问题研究综述. 水利学报, 2006, 37: 141-149

37 Yu G R, Nakayama K, Matsuoka N, et al. A combination model for estimating stomatal conductance of maize (Zea mays L.) Leaves over a long term. Agr Forest Meteorol, 1998, 92: 9-28

38 Egea G, Verhoef A, Vidale P L. Towards an improved and more flexible representation of water stress in coupled photosynthesis-stomatal conductance models. Agr Forest Meteorol, 2011, 151: 1370-1384

39 Zhang B Z, Liu Y, Xu D, et al. Evapotranspiraton estimation based on scaling up from leaf stomatal conductance to canopy conductance. Agr Forest Meteorol, 2011, 151: 1086-1095

40 Rochette P, Pattey E, Desjardins R L, et al. Estimation of maize (Zea mays L.) Canopy conductance by scaling up leaf stomatal conductance. Agr Forest Meteorol, 1991, 54: 241-261 